\title{
Quality Inspection System for Explosive Actuated Devices
}

\author{
Chunlei Tian, Zhifeng Fan, Chen Li
}

\author{
Shijiazhuang Mechanical Engineering College, Shijiazhuang, China
}

\begin{abstract}
Keywords: Explosive actuated devices; Quality inspection system; Performance testing.
\end{abstract}
\begin{abstract}
Explosive actuated device is one kind of electric-explosive initiator often be used in weapon and aviation and space flight equipments' executing system. As one of the important firing units in explosive chain, its quality affects the safety and reliability of whole system greatly. Therefore, when using actuated device, the performance of the each batch of products needs to be tested exactly. At present, the equipments being used to test this character are very simple, and their testing means, process and results no longer meet the needs. By using the technologies of virtual instrument and the digital electronic measurement, a test device was demonstrated, designed and manufactured. The proposed system is different from the existing ones by featuring computer control, convenience in use, high precision of parameters setting, automatic data processing, management and judgment.
\end{abstract}

\section{Introduction}

Explosive actuated device is an independent unit, filled with energy materials as black powder and high explosives, which usually can be initiated by electric energy to burn or explode and translate its chemical energy to the form of mechanical work to achieve desired function. As a key to release the energy of explosives, the actuated device is an indispensable part to any explosive control system, which provides the finally action energy for desired movement, such as parts separate, rope cutting, piston shift and etc. For its high reliability, powerful, convenience in usage, safety in storage and transportation, explosive actuated device is used in common ammunition, missiles, nuclear weapons, and aviation and space flight fields for many years.

Although the explosive actuated device is small in size, light in weight and simple in structure, its performance and quality have a direct bearing on the safety and reliability of whole explosive chain work effect greatly. A faulty actuated device may lead to disastrous effect. For the limitation of producing condition, many actuated devices' quality is hard to control. The differences of character parameters of same type of products made in same plant are existed usually. Therefore, when using this kind of explosive devices, the performance of the individual batch of products needs to be tested exactly.

At present, the equipments being used to test the quality for explosive actuated devices are very simple; most of them testing process were controlled by manual, and cannot get all useful parameter in one test process. The precision of result and the working efficiency no longer meet the needs. The author designed a new quality inspection system of explosive actuated by using the technologies of virtual instrument, computer control, and digital electronic measurement technology.

\section{Testing parameters select}

\subsection{Working theory of explosive actuated device}

For different purpose, there are many kinds of actuated device and their structure is dissimilarly. Most of the products have two leg wires embedded in a metal shell which contains high explosive base charge designed to do mechanical work. Sketch map of actuated device is shown in Figure 1.

Electric-explosive actuated devices are typically designed with an ignition mixture, a pyrotechnic fuse train (for the delay element) and a base charge, respectively. A thin metal filament, known as a bridge-wire, is attached between each end of the leg wire and is embedded in an ignition mixture. The 
pyrotechnic delay element is designed to burn at an approximated rate. The length and composition of the pyrotechnic train control the approximate rate of burn and thus the timing when the initiator fires.

When sufficient electrical current passes through the bridge wire, it becomes hot enough to ignite the ignition mixture. This event initiates the pyrotechnic element in the delay train which then initiates the base charge. Then the charge emits lots of hot gases and huge heat instantaneous. With the expanding of the hot gases, the action parts are pushed move in designed way and the scheduled function is achieved finally.

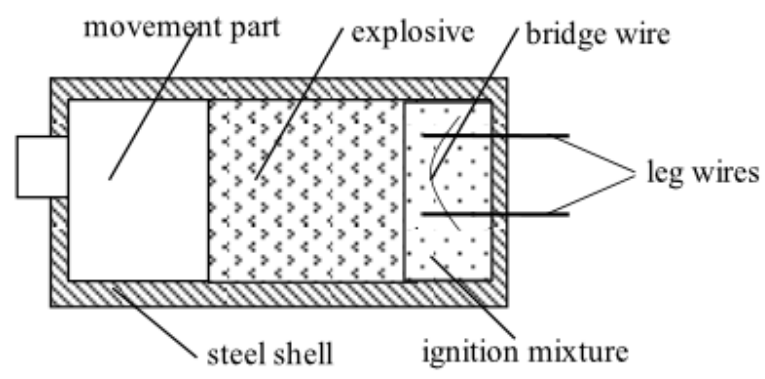

Figure 1. Sketch map of actuated device

\subsection{Testing parameter select}

Since explosive actuated device are designed to fire when electrical energy is supplied to them, any extraneous source of electric current represents a potential source for initiation. The resistance of bridge-wire effect the initiate energy amount and the initiate time greatly when the detonator fires. When using electric initiators, the continuity and resistance of the individual product needs to be tested exactly.

When electric actuated device is initiated, current leakage from the initiating circuit must also be prevented. If bare wires are allowed to come into contact with another conductor or even a conductive portion of the shell, some of the electric energy may leak out of the circuit causing misfires.

We named the time between electric current inputs to the base charge fired entirety is delay time. That process is controlled by the performance of delay element contained in tested actuated device, which is an important quality index of the product.

The amount of produced hot gases and emitted heat is the energy source of finally mechanical work. The speed of gases expands affect the ability to perform or act effectively.

From above, we select resistance of bridge-wire, insulating resistance between electric poles and external tube, delay time, pressure-time curve, distance of movement part of device as the main performance to judge explosive actuated device's quality.

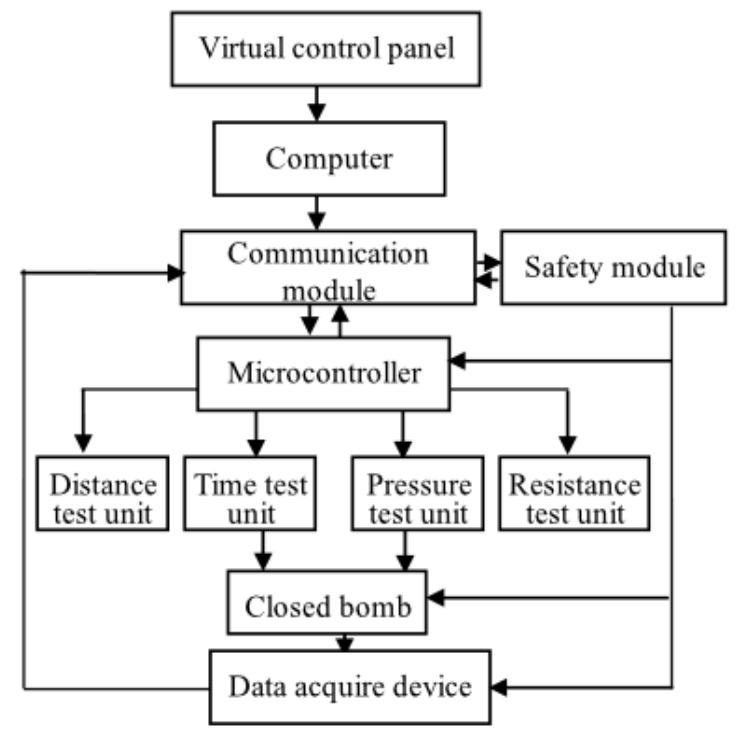

Figure 2. Hardware structure of testing system 


\section{Structure of hardware}

The whole quality inspection system is consists of computer, digital powder, communication module, digital data acquire module, safety module, closed bomb, resistance testing module, pressure testing module, time testing module and distance testing module. The diagram of test systems is shown as Figure 2. The function of each part as follows.

\subsection{Computer}

Computer is the key of whole hardware system, which connected with the external devices by communication channels. The function of this computer including communication with 1394 interface, manages the resource of self, executes the test procedure, processing the received data, judging and giving out the quality condition of each product.

\subsection{Digital Data Acquire Module}

This module is consists of multimeter and A/D device. Multimeter is used to measure the voltage and current at certain point of the testing circuit or the signals send by sensors. With the help of computer and A/D device, needed results will be shown in digital form and then send to computer.

\subsection{Safety Module}

The function of safety model is to surveillance the working condition of whole system. Safety model is including testing room and inspecting circuits. The task of inspecting circuits is checking the working condition of each part. If something is abnormal during test, the alarm will be ring and emergency safety procedure will be done immediately.

\subsection{Closed Bomb}

This part is testing room, which made of steel, is the container of the initiator during the testing process. There is a seat for fixing the testing device in the center of the bomb. Sensor of each testing module be fixed around the testing device in the bomb.

\subsection{Measure Resistance}

We use DC bridge to test the bridge-wire resistance value, and use constant current source to get the range of the insulating resistance. This two function circuits can be selected with the help of multi-switch under the control of the computer.

\subsection{Measure Gas Pressure}

We use piezoelectric effect to measuring pressure of the gas in bomb. The rang of selected piezoelectric sensor from $0 \mathrm{Mpa}$ to 600Mpa. The signals gated by sensor send to computer after filtered and magnified in time.

Except that, electromagnetic sensor be used record the distance signal, electric probe catch timer start or stop single, digital powder supply power to each part of the system, and the microcontroller help the computer executes the test procedure.

\section{Made up of software}

Software is the platform for testing system to actualize its function. Now people often use different tools to write application programs for testing system. The famous one is Lab Windows/CVI, which provide by NI Company. If you grasp language $C$ well enough, you can use ANSI $C$ to write your system freely. The other is Lab View and HP VEE. The latter is provided by HP Company. They all use graphics language to write application program. After select needed instrument icons and set parameters correctly and connect the icons in order, the whole program will be formed immediately.

The process of main program is shown in Fig.3.

Our system is written with Lab View based Windows XP operating system. User interface is good and the whole system consists of different function modules. Each test item can be finished in one appointed particular module. This way not only improved the reliability of system but also lowered the demand of skill for system user obviously. Except that, users can modify or add any other unit by their own purpose easily and cheaply. 
When testing program is starting, system self-check module will work at first. Only on the condition of everything is ok that each system parameter begins to initialize. According to the type of the testing sample, working condition and parameters can de adjust according to the need of operator in performance setting module. Then click the start button, the resistance of bridge-wire and insulating resistance will be checked at first. And then, the actuated device will be fired after the delay time, pressure curve, moving speed and distance data will be recorded in computer at the same time. According to the technique standard, the quality of the product will be judged by software automatically directly at last.

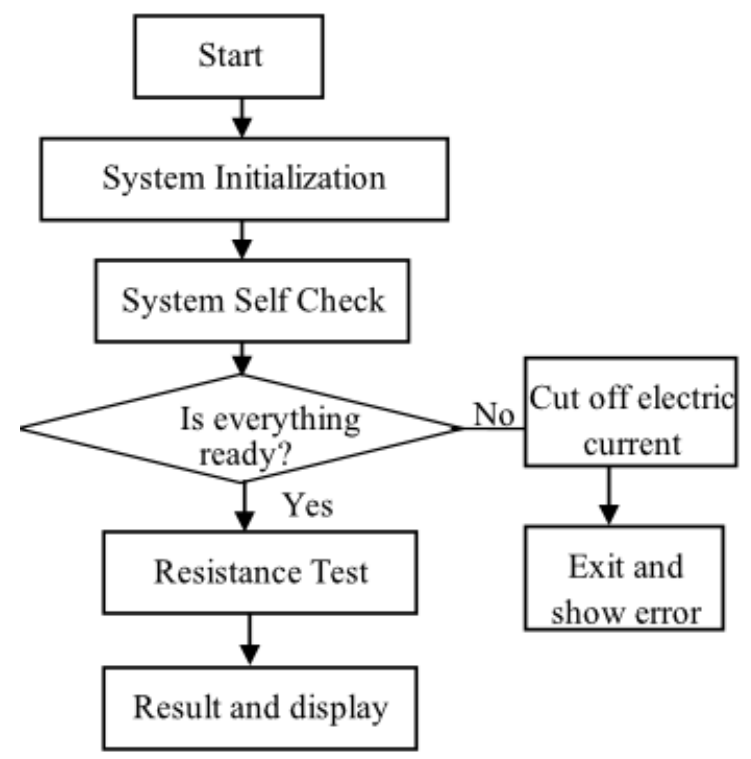

Figure 3. Main program of testing system

During the testing process, safety module inspects the condition of initiator and working condition of the system all the time. If something is abnormal, the current through the initiator will be cut off and the procedure will be exit immediately.

Testing data, final result, testing process and working condition of system can be shown in display device isochronously. All data can be stored as record in database, which is managed by Microsoft access system.

\section{Summary}

The explosive actuated device quality inspects system consist of two parts. The one part is hardwires, which made up of steel closed bomb, sensors, measure circuit, and computer. The other part is software, which combined with modem computer science and virtual instrument testing technology. With the help of this system, the quality test work can be done easily. From above we can say that this system not only can be widely used in military plant but also can be popularized in other demolition fields. Compared with traditional testing device, this system has following advantages.

a. Being used computer auto check technology, the working efficiency and result veracity improved markedly.

b. All system consists of different modules, the following maintenance and extension work can to be carried out easily.

c. Structure of this system is simple, which improved the mobility of testing device.

d. Being added safety module and backup circuits, the safety and reliability is improved greatly.

\section{References}

[1] Bi Wen-hui \& He Liu-shuang. 2008. Study on the forhead with a wrapper or bridgewire. Initiators \& Pyrotechnics. (3):15-17. 
[2] Chen Sheng-wen \& Cheng Yi. 2002. The influence of the coherence of bridgewire on the reliability of the electric igniter study. Initiators \& Pyrotechnics. (4):15-17.

[3] Huang You-rui \& Hu Yi-hua. 2003. Development of nondestructive measuremetal instrument of EED. Journal of Electronic Measurement and Instrument. (4):63-66.

[4] Huang Zheng-ping \& He Yuan-hang. 2005. Explosion measurement techniques, Beijing Institute Technology Press: Beijing.

[5] Jin Xiao-li \& Zhao xiao-bin. 2004. Study on a new type measurement system of closed bomb. Initiators \& Pyrotechnics. (3):44-46.

[6] LI Guo-xin \& CHENG Guo-xin. 2004. Experiment and testing technology of explosive initiator, Beijing Institute Technology Press: Beijing.

[7] Mu Li-jun \& Gao Jun-guo. 2009. The influence of the coherence of bridgewire on the reliability of the electric igniter study. Journal of Sichuan Ordance.30 (4):23-26.

[8] WANG Kai-min. 2014. Engineering of initiator \& pyrotechnics, National Defense Industry Press: Beijing.

[9] WANG Kai-min \& WEN Yu-quan. 2006. Design of initiators and pyrotechnics for weapon system, National Defense Industry Press: Beijing.

[10]Zhou Shi-zheng. 2002. Virtual instrument and virtual instrument development workbench. Journal of Chengdu petroleum college. 2(2):23-25. 